\title{
ASIC Implementation Comparison of SIC and LSD Receivers for MIMO-OFDM
}

\author{
Johanna Ketonen, Markus Myllylä and Markku Juntti \\ Centre for Wireless Communications \\ P.O. Box 4500, FIN-90014 University of Oulu, Finland \\ \{johanna.ketonen, markus.myllyla, markku.juntti\}@ee.oulu.fi
}

\author{
Joseph R. Cavallaro \\ Dept. of Electrical \& Computer Engineering \\ Rice University, Houston, TX 77251-1892, USA \\ cavallar@rice.edu
}

\begin{abstract}
MIMO-OFDM receivers with horizontal encoding are considered in this paper. The successive interference cancellation (SIC) algorithm is compared to the $\mathrm{K}$-best list sphere detector (LSD). A modification to the K-best LSD algorithm is introduced. The SIC and K-best LSD receivers are designed for a $2 \times 2$ antenna system with 64-quadrature amplitude modulation (QAM). The ASIC implementation results for both architectures are presented. The K-best LSD outperforms the SIC receiver in bad channel conditions but the SIC receiver performs better in channels with less correlated MIMO streams. The latency of the K-best LSD is large due to the high modulation order and list size. The throughput of the SIC receiver is more than 6 times higher than that of the K-best LSD.
\end{abstract}

\section{INTRODUCTION}

Multiple-input multiple-output (MIMO) systems offer an increase in capacity or diversity. Orthogonal frequency division multiplexing (OFDM) is a popular technique for wireless high data-rate transmission because it enables efficient use of the available bandwidth and a simple implementation. The combination of MIMO and OFDM is a promising wireless access scheme [1]. Successive interference cancellation (SIC) for third generation (3G) long term evolution (LTE) MIMOOFDM systems is considered in this paper. The 3G LTE standard includes a downlink transmitter structure, where the data is divided into two streams which are encoded separately [2]. Therefore, a decoded layer can be used in interference cancellation.

Instead of jointly detecting signals from all the antennas, the strongest signal can be detected first and its interference can be cancelled from each received signal [3]. In channel coded systems, the detected symbols are decoded before cancellation. The soft bit decisions from the turbo decoder are used to calculate symbol expectations. The expectations are cancelled from the remaining layers.

Sphere detectors calculate the maximum likelihood (ML) solution by taking into account only the lattice points that are inside a sphere of a given radius [4]. List sphere detectors (LSD) approximate the maximum a posteriori probability (MAP) detector and provide soft outputs for the decoder [5]. The K-best LSD algorithm is a modification of the K-best algorithm [6]. The K-best algorithm has been implemented in

This research was financially supported in part by Tekes, the Finnish Funding Agency for Technology and Innovation, Nokia, Texas Instruments, Nokia Siemens Networks and Elektrobit. a $4 \times 4$ antenna system with 16-QAM for uncoded system [7] and a soft-output version for coded system [8].

In this paper, the complexity and latency of the the iterative linear minimum mean square error (LMMSE) based SIC receiver is studied and compared to those of the K-best LSD receiver. The impact of word lengths on the performance and complexity is also considered. The ASIC implementation results are obtained with the Catapult Synthesis tool [9], which generates register transfer language (RTL) from $\mathrm{C}$ code. The K-best LSD and SIC receivers are designed for $2 \times 264$ QAM system and gate counts of the ASIC implementations are presented. Their feasibility for a real $3 \mathrm{G}$ LTE system is discussed.

The SIC and K-best LSD implementations for a field programmable gate array (FPGA) are compared in [10]. The receivers were designed for quadrature phase shift keying (QPSK), 16-QAM and 64-QAM and implemented with the Xilinx System Generator. The SIC receiver was found to be slightly more complex than the K-best LSD receiver. However, the latency of the SIC receiver was lower with all modulations.

The paper is organized as follows. The system model is presented in Section II. The SIC algorithm is introduced in Section III and the K-best LSD algorithm in Section IV. Some performance examples are shown in Section V. The implementation results and latencies are compared in Section VI. Conclusions are drawn in Section VII.

\section{System MODEL}

An OFDM based MIMO transmission system with $N$ transmit (TX) and $M$ receive (RX) antennas, where $N \leq M$, is considered in this paper. A layered space-time architecture with horizontal encoding is applied. The system model is illustrated in Figure 1. The data is divided into two streams which are encoded separately. The encoded data is interleaved, modulated and mapped to different antennas. In the receiver, the received signal is detected jointly or separately, loglikelihood ratios (LLR) are created from the detected symbols which are then deinterleaved. Decoding is also performed separately.

The received signal can be described as

$$
\mathbf{y}_{p}=\mathbf{H}_{p} \mathbf{x}_{p}+\eta_{p}, \quad p=1,2, \ldots, P,
$$




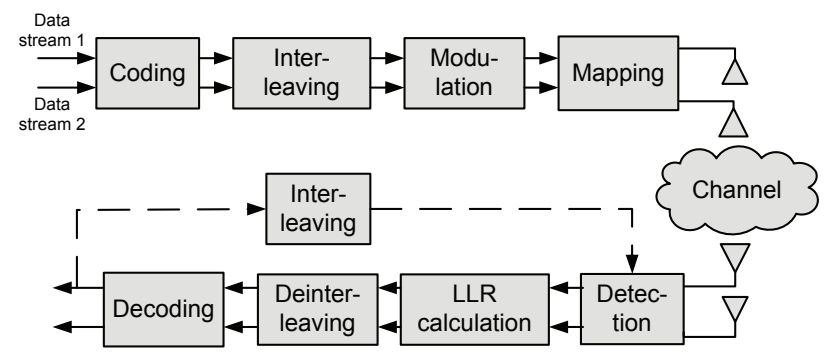

Fig. 1. The MIMO-OFDM system model in 3G LTE.

where $P$ is the number of subcarriers, $\mathbf{x}_{p} \in \mathbb{C}^{N \times 1}$ is the transmitted signal on $p$ th subcarrier, $\eta_{p} \in \mathbb{C}^{M \times 1}$ is a vector containing complex Gaussian noise with variance $\sigma^{2}$ and $\mathbf{H}_{p} \in \mathbb{C}^{M \times N}$ is the channel matrix containing complex Gaussian fading coefficients. The entries of $\mathbf{x}_{p}$ are from a complex QAM constellation $\Omega$ and $|\Omega|=2^{Q}$, where $Q$ is the number of bits per symbol. The set of possible transmitted symbol vectors is $\Omega^{N}$.

\section{THE SIC ALgORITHM}

The soft SIC receiver is illustrated in Figure 2. The first layer is detected with a LMMSE detector. The scaling block calculates log-likelihood ratio (LLR) values from the LMMSE outputs. The de-interleaved stream is decoded with a turbo decoder and symbol expectations are calculated. The expectations are cancelled from the second layer. The first layer remains the same after the second iteration.

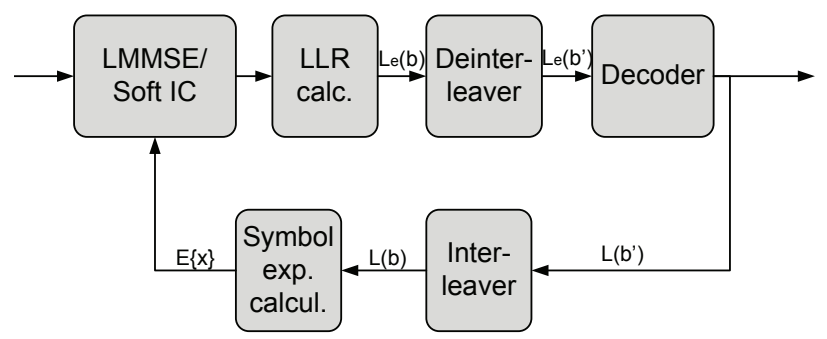

Fig. 2. Structure the soft IC receiver.

The weight matrix is calculated with MMSE algorithm

$$
\mathbf{W}=\left(\mathbf{H}^{\mathrm{H}} \mathbf{H}+\sigma^{2} \mathbf{I}_{M}\right)^{-1} \mathbf{H}^{\mathrm{H}},
$$

where $\mathbf{H}$ is the channel matrix, $\sigma^{2}$ is the noise variance, $(\cdot)^{\mathrm{H}}$ is the complex conjugate transpose and $\mathbf{I}_{M}$ is a $M \times M$ identity matrix. The layer for detection is chosen according to the postdetection signal-to-noise-plus-interference ratio (SNIR) and the corresponding nulling vector is chosen from the weight matrix $\mathbf{W}$ [3]. All the weight matrices in an OFDM symbol are calculated and layer to be detected is chosen according to the average over all the subcarriers.

The LLRs are calculated from the LMMSE outputs as presented in [11]. The symbol expectation calculation is sim- plified from

$$
E\{x\}=\left(\frac{1}{2}\right)^{k} \sum_{x^{\prime} \in \Omega} x^{\prime} \prod_{i=1}^{k}\left(1+b_{i} \tanh \left(\log \mathrm{P}\left\{c_{i}\right\} / 2\right)\right),
$$

where $\log \left\{c_{i}\right\}$ are the LLRs of coded bits corresponding to $x, b_{i}$ are bits corresponding to constellation point $x^{\prime}, \Omega$ is the symbol alphabet and $k$ is the number of bits per symbol, into one tangent calculation in real and imaginary parts of the symbol expectation

$$
E\{x\}_{\mathrm{re}}=\operatorname{sgn}\left(\left(\log P_{i}\right) S\left|\tanh \left(\log P_{i+2}\right)\right| .\right.
$$

The constellation point $S$ is chosen to be 1,3,5 or 7 depending on the signs of $\log P_{i+1}$ and $\log P_{i+2}$.

\section{The K-BEST LSD ALgORITHM}

List sphere detectors can be used to approximate the MAP detector and to provide soft outputs for the decoder [5]. The sphere detector (SD) algorithms solve the ML solution with a reduced number of considered candidate symbol vectors. They take into account only the lattice points that are inside a sphere of a given radius. The condition that the lattice point lies inside the sphere can be written as

$$
\|\mathbf{y}-\mathbf{H x}\|^{2} \leq C_{0}
$$

After QR decomposition (QRD) of the channel matrix $\mathbf{H}$ in (5), it can be rewritten as

$$
\left\|\mathbf{y}^{\prime}-\mathbf{R x}\right\|^{2} \leq C_{0}^{\prime}
$$

where $C_{0}^{\prime}=C_{0}-\left\|\left(\mathbf{Q}^{\prime}\right)^{\mathrm{H}} \mathbf{y}\right\|^{2}, \mathbf{y}^{\prime}=\mathbf{Q}^{\mathrm{H}} \mathbf{y}, \mathbf{R} \in \mathbb{C}^{N \times N}$ is an upper triangular matrix with positive diagonal elements, $\mathbf{Q} \in \mathbb{C}^{M \times N}$ is a matrix with orthogonal columns and $\mathbf{Q}^{\prime} \in$ $\mathbb{C}^{M \times(M-N)}$ is a matrix with orthogonal columns.

The squared partial Euclidean distance (PED) of $\mathbf{x}_{i}^{N}$, i.e., the square of the distance between the partial candidate symbol vector and the partial received vector, can be calculated as

$$
d\left(\mathbf{x}_{i}^{N}\right)=\sum_{j=i}^{N}\left|y_{j}^{\prime}-\sum_{l=j}^{N} r_{j, l} x_{l}\right|^{2},
$$

where $i=N \ldots, 1$ and $\mathbf{x}_{i}^{N}$ denotes the last $N-i+1$ components of vector $\mathbf{x}[4]$.

The K-best algorithm [6] is a breadth-first search based algorithm, and keeps the $K$ nodes which have the smallest accumulated Euclidean distances at each level. If the PED is greater than the squared sphere radius $C_{0}$, the corresponding node will not be expanded.

A LSD structure is illustrated in Figure 3. The channel matrix $\mathbf{H}$ is first decomposed to matrices $\mathbf{Q}$ and $\mathbf{R}$ in the QR-decomposition block. Euclidean distances between the receiver signal vector $\mathbf{y}$ and possible transmitted symbol vectors are calculated in the LSD block. The candidate symbol list is demapped to binary form. The log-likelihood ratios are calculated in the LLR block. Limiting the range of LLRs reduces the required list size $K$ [12]. 


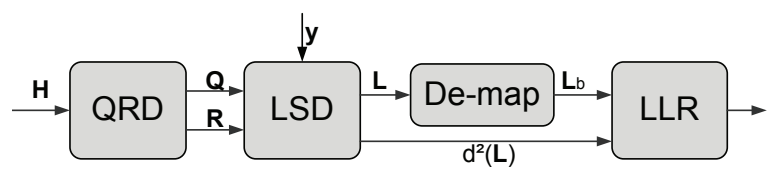

Fig. 3. Structure of the LSD receiver.

The breadth-first tree search can be modified to decrease the latency. Two PEDs are calculated in parallel and the larger one is discarded. With 64-QAM, instead of having to sort 64 PEDs, there are only 32 PEDs to be sorted on each level. On the first level, PEDs are calculated as with the original breadth-first search as shown in Figure 4, where the nodes with grey paths are discarded.



Fig. 4. The modified tree search.

\section{Performance Comparison}

A 3G LTE [2] based MIMO-OFDM system model was assumed in the simulations. A $2 \times 2$ antenna system with 64QAM was applied along with turbo coding with $1 / 2$ code rate, horizontal encoding and a $5 \mathrm{MHz}$ bandwidth with 512 subcarriers (300 used). A 6-tap typical urban (TU) and a 20tap Winner B1 channel model with a $120 \mathrm{kmph}$ user velocity was assumed.

The performance of the K-best LSD and the SIC receiver can be seen in Figure 5, where frame error rates (FER) vs. signal to noise ratio (SNR) are presented. The simulations were performed also with fixed-point arithmetic. The K-best LSD was simulated with 16 and 12 bit word lengths and list sizes 8 and 16. The SIC simulations were performed with optimized low complexity LLR and expectation calculation. The channel in Figure 5 is highly correlated and has a large delay spread. It can be seen that the SIC receiver performs worse than the LSD. The SIC receiver outperforms the LSD in better channel conditions as illustrated in Figure 6. In good channel conditions, the SIC receiver successfully cancels the interference from the second layer and the list size in the Kbest LSD is not large enough to achieve the SIC performance. When the channel conditions are bad, there are more errors in the detection of the first layer, which leads to error propagation in the cancellation.

The impact of the modified search on the FER is shown in Figure 7 . It can be seen that the performance degradation is minimal.

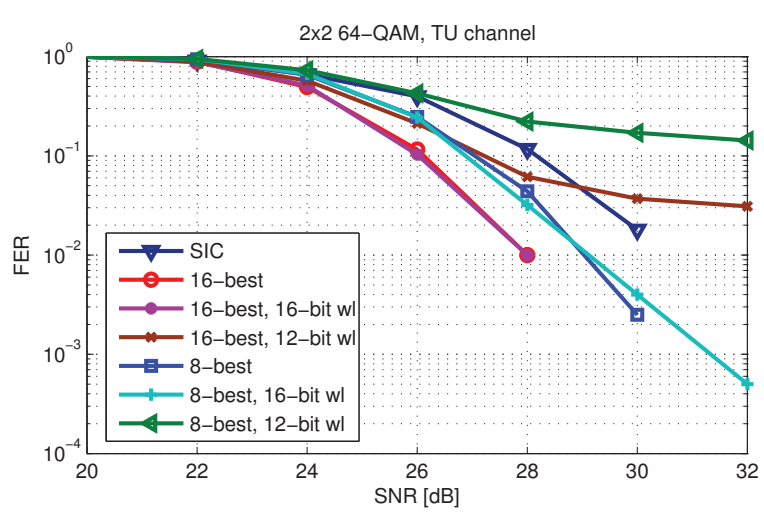

Fig. 5. K-best LSD vs. SIC in TU channel.

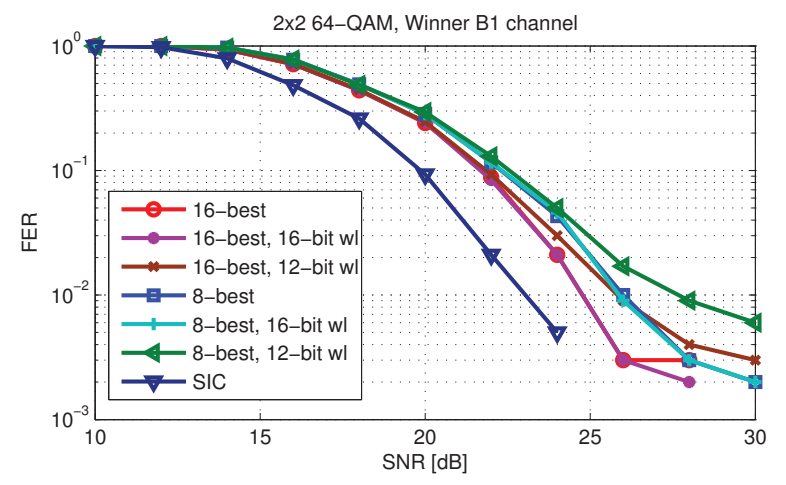

Fig. 6. K-best LSD vs. SIC in Winner B1 channel.

\section{IMPLEMENTATION RESULTS}

\section{A. K-best LSD Receiver}

The QR-decomposition was based on the squared Givens rotations (SGR). The K-best LSD architecture consists of four PED calculation blocks, i.e., one block for each layer and three sorters. The architecture is shown in Figure 8.

The architecture of the second stage partial Euclidean distance calculation and sorting with the modified K-best LSD is illustrated in Figure 9. The sorter is a parallel insertion sorter [13]. The PED is compared to all previous PEDs stored in the register. If the PED is smaller than a value stored in the register, the PED is inserted in to the corresponding slot. The values larger than the inserted PED are shifted while the largest value is dropped out. After all the PEDs have been sorted, the $K$ best values are shifted to another register and the next symbol can be processed. The sorters have 8 or 16 registers depending on the list size.

Catapult $($ C C Synthesis tool [9] was used in the implementation of the receivers. It synthesizes algorithms written in ANSI C++ into high-performance, concurrent hardware. This single source methodology allows designers to pick the best architecture for a given performance/area/power specification while minimizing design errors and reducing the overall verification burden. 


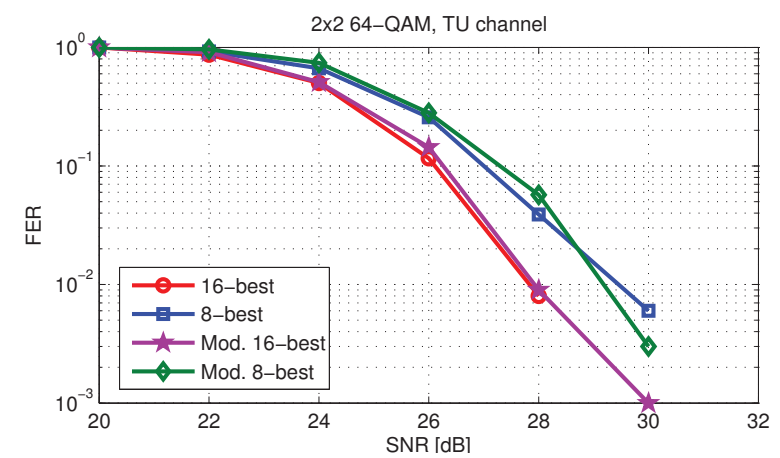

Fig. 7. Impact of modified tree search on performance.

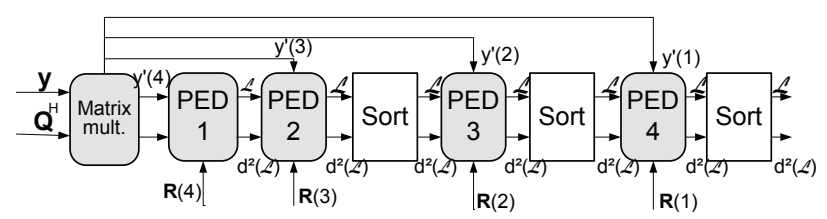

Fig. 8. The structure of the K-best LSD.

The QRD, de-mapping and LLR calculation blocks from the K-best LSD receiver were implemented with Catapult Synthesis tool. The K-best LSD block was hand-coded with VHDL. The implementation results can be seen in Table I, where the number of equivalent gates, the clock frequency and the throughput period of 12 bits in clock cycles are presented. The complexity of the K-best LSD with a list size 16 is twice of the complexity with a list size 8 . The latency is also twice as large. The total number of gates include the $\mathrm{QRD}$, the original K-best LSD and the LLR blocks. The modified K-best adds 10 $\mathrm{k}$ gates to the complexity of the original K-best but it doubles the throughput.

If the QRD is calculated only when the channel realization changes, the $\mathbf{Q}$ and $\mathbf{R}$ matrices have to be stored in a memory. For a $5 \mathrm{MHz}$ bandwidth, i.e., 300 subcarriers, 94 kbit of memory is needed. However, since the latency of the QRD block is low, QR-decomposition could be performed for every channel realization, obviating the need for memory.

TABLE I

THE K-BEST LSD RECEIVER IMPLEMENTATION RESULTS

\begin{tabular}{|l|l|l|l|}
\hline Block & Area (GE) & Clock freq. & Tp. period \\
\hline QRD & $61 \mathrm{k}$ & $100 \mathrm{MHz}$ & 4 \\
\hline K-best LSD, K =8 & $52 \mathrm{k}$ & $100 \mathrm{MHz}$ & 64 \\
\hline K-best LSD, K =16 & $99 \mathrm{k}$ & $100 \mathrm{MHz}$ & 128 \\
\hline Mod. K-best LSD, K =8 & $62 \mathrm{k}$ & $100 \mathrm{MHz}$ & 32 \\
\hline Mod. K-best LSD, K =16 & $109 \mathrm{k}$ & $100 \mathrm{MHz}$ & 64 \\
\hline Mod. K-best LSD, K =8 & $68 \mathrm{k}$ & $200 \mathrm{MHz}$ & 32 \\
\hline LLR calculation, K =16 & $31 \mathrm{k}$ & $100 \mathrm{MHz}$ & 16 \\
\hline Total, K=8 & $144 \mathrm{k}$ & $100 \mathrm{MHz}$ & 64 \\
\hline Total, K=16 & $191 \mathrm{k}$ & $100 \mathrm{MHz}$ & 128 \\
\hline
\end{tabular}

\section{B. SIC Receiver}

The architecture of symbol expectation calculation is depicted in Figure 10. The lookup table (LUT) is used to get the



Fig. 9. Partial Euclidean distance calculation and sorting.

value of $\left|\tanh \left(\log P_{i+2}\right)\right|$ from (4). The imaginary part of the expectation is calculated in parallel with the real part from the next three bit LLRs.

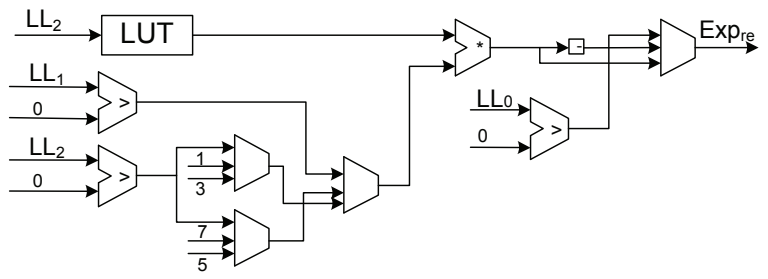

Fig. 10. The symbol expectation calculation architecture.

The LMMSE, the LLR calculation, the symbol expectation calculation and interference cancellation blocks from the SIC receiver were also be implemented with the Catapult $\mathrm{C}$ tool. The implementation results are shown in Table II. The LMMSE is also based on the SGR and the block has the highest throughput period. The LLR calculation block calculates 6 LLRs in one clock cycle. Also symbol expectations from 6 LLRs are calculated in one clock cycle. The channel matrix and the received symbol vector have to be stored in memory. The memory requirement with 300 subcarriers is 57.6 kbits.

TABLE II

THE SIC RECEIVER IMPLEMENTATION RESULTS

\begin{tabular}{|l|l|l|l|}
\hline Block & Area (GE) & Clock freq. & Tp. period \\
\hline LMMSE & $168 \mathrm{k}$ & $100 \mathrm{MHz}$ & 8 \\
\hline LLR calculation & $30 \mathrm{k}$ & $100 \mathrm{MHz}$ & 1 \\
\hline Symbol exp. & 1890 & $100 \mathrm{MHz}$ & 1 \\
\hline SIC & $28 \mathrm{k}$ & $100 \mathrm{MHz}$ & 1 \\
\hline Total & $229 \mathrm{k}$ & $100 \mathrm{MHz}$ & \\
\hline
\end{tabular}

\section{Latencies}

The latency of a receiver can be expressed as

$$
D_{\text {rec }}=D_{\text {det }}+\left(D_{\text {LLR }}+D_{\text {dec }}\right) \times N_{\text {iter }},
$$

where $D_{\text {det }}$ is the latency of the detector, $D_{\text {LLR }}$ is the latency of LLR calculation, $D_{\mathrm{dec}}$ is the latency of the decoder and $N_{\text {iter }}$ is the number of iterations. The throughput of a receiver can be calculated as

$$
\frac{1}{D_{\text {rec }}} \times Q \times N
$$

where $Q$ is the number of bits per symbol. 
The LMMSE weight matrices are calculated only every 7 OFDM symbols, i.e., it is assumed that the channel stays the same for $0.5 \mathrm{~ms}$. The SIC receiver has to calculate all the LMMSE weight matrices in an OFDM symbol before the decision on the better layer can be made. It also has to wait for the decoder to finish decoding the first layer before the symbols estimates can be calculated. A high throughput turbo decoder [14] was used in estimating the throughput of the SIC receiver.

The latencies of the K-best LSD receiver are presented in Table III and the SIC receiver in Table IV. Two iterations are performed in the SIC receiver. The throughput period is $48.3 \mathrm{~ns}$ and the throughput of the SIC receiver is then 248 $\mathrm{Mb} / \mathrm{s}$. The K-best LSD receiver is not iterative. Therefore, only the latency $D_{\text {det }}$ is taken into account in the throughput calculations. The throughput period of the modified K-best LSD receiver is $0.32 \mu \mathrm{s}$ and the throughput is $37.5 \mathrm{Mb} / \mathrm{s}$. The SIC receiver is then more than 6 times faster than the K-best LSD. The throughput of the original K-best LSD with list size 8 is $18.75 \mathrm{Mb} / \mathrm{s}$. A $75 \mathrm{Mb} / \mathrm{s}$ throughput can be achieved with a $200 \mathrm{MHz}$ clock frequency and the modified 8-best LSD.

TABLE III

THE K-BEST LSD RECEIVER LATENCIES

\begin{tabular}{|l|l|l|}
\hline Block & Latency & Tp. period \\
\hline QRD & $0.19 \mu \mathrm{s}$ & $40 \mathrm{~ns}$ \\
\hline K-best LSD, K =8 & $2.06 \mu \mathrm{s}$ & $0.64 \mu \mathrm{s}$ \\
\hline K-best LSD, K =16 & $4.1 \mu \mathrm{s}$ & $1.28 \mu \mathrm{s}$ \\
\hline Mod. K-best LSD, $\mathrm{K}=8$ & $1.17 \mu \mathrm{s}$ & $0.32 \mu \mathrm{s}$ \\
\hline Mod. K-best LSD, K =16 & $2.14 \mu \mathrm{s}$ & $0.64 \mu \mathrm{s}$ \\
\hline LLR calculation & $0.29 \mu \mathrm{s}$ & $0.16 \mu \mathrm{s}$ \\
\hline Total (Mod. K-best), K=8 & $1.65 \mu \mathrm{s}$ & $0.32 \mu \mathrm{s}$ \\
\hline
\end{tabular}

TABLE IV

THE SIC RECEIVER LATENCIES

\begin{tabular}{|l|l|l|}
\hline Block & Latency & Tp. period \\
\hline LMMSE & $0.38 \mu \mathrm{s}$ & $80 \mathrm{~ns}$ \\
\hline LLR calculation & $80 \mathrm{~ns}$ & $10 \mathrm{~ns}$ \\
\hline Symbol exp. & $80 \mathrm{~ns}$ & $10 \mathrm{~ns}$ \\
\hline SIC & $20 \mathrm{~ns}$ & $10 \mathrm{~ns}$ \\
\hline Turbo decoder [14] & & $16.87 \mathrm{~ns}$ \\
\hline Total & $0.64 \mu \mathrm{s}$ & $31.5 \mathrm{~ns}$ \\
\hline Total (with decoder) & $0.66 \mu \mathrm{s}$ & $48.3 \mathrm{~ns}$ \\
\hline
\end{tabular}

According to the $3 \mathrm{G}$ LTE specifications, the maximum time frame for processing an OFDM symbol is $83.3 \mu \mathrm{s}$. In a $5 \mathrm{MHz}$ bandwidth, there are 300 used subcarriers. The SIC receiver processes 300 subcarriers in $14.5 \mu$ s. The SIC receiver could also be used in a $20 \mathrm{MHz}$ bandwidth, where the processing of 1200 subcarriers would take $58 \mu$ s. The K-best LSD receiver can not process the subcarriers in the required time. Only the modified K-best with a list size 8 and a $200 \mathrm{MHz}$ clock frequency would process 300 subcarriers in $48 \mu$ s.

\section{CONCLuSions}

The performance, complexity and latency of the K-best LSD and the SIC receivers was compared. A modification to the K-best LSD tree search was introduced. It doubles the throughput compared to the original K-best LSD with minimal performance degradation. The receivers were designed for a $2 \times 2$ antenna system and 64-QAM. The performance of the SIC receiver is worse than that of the K-best LSD in a correlated channel but the SIC receiver performance is better when the MIMO streams are less correlated. The complexity of the SIC receiver is higher than the complexity of the Kbest LSD. The throughput of the SIC receiver is $248 \mathrm{Mb} / \mathrm{s}$ and the throughput of the K-best LSD receiver is $37.5 \mathrm{Mb} / \mathrm{s}$. The throughput of the SIC receiver is more than 6 times higher than that of the K-best LSD receiver as the latency of the $\mathrm{K}$-best LSD increases with the modulation and list size.

\section{ACKNOWLEDGMENTS}

The authors would like to thank Mentor Graphics for the possibility to evaluate Catapult C (B) Synthesis tool.

\section{REFERENCES}

[1] H. Bölcskei and E. Zurich, "MIMO-OFDM wireless systems: basics, perspectives, and challenges," IEEE Wireless Communications, vol. 13, no. 4, pp. 31-37, August 2006.

[2] 3rd Generation Partnership Project (3GPP); Technical Specification Group Radio Access Network, "Evolved universal terrestrial radio access E-UTRA; LTE physical layer TS 36.201 version 8.1.0)," Tech. Rep., 2007.

[3] P. W. Wolniansky, G. J. Foschini, G. D. Golden, and R. A. Valenzuela, "V-BLAST: An architecture for realizing very high data rates over the rich-scattering wireless channel," in International Symposium on Signals, Systems, and Electronics (ISSSE), Pisa, Italy, Sep. 29-Oct. 2 1998, pp. 295-300.

[4] M. O. Damen, H. E. Gamal, and G. Caire, "On maximum-likelihood detection and the search for the closest lattice point," IEEE Transactions on Information Theory, vol. 49, no. 10, pp. 2389-2402, October 2003.

[5] B. Hochwald and S. ten Brink, "Achieving near-capacity on a multipleantenna channel," IEEE Transactions on Communications, vol. 51, no. 3 , pp. 389-399, March 2003.

[6] K. Wong, C. Tsui, R.-K. Cheng, and W. Mow, "A VLSI Architecture of a K-best Lattice Decoding Algorithm for MIMO Channels," in Proc. IEEE Int. Symp. Circuits and Systems, vol. 3, Helsinki, Finland, June 2002, pp. 273-276.

[7] M. Wenk, M. Zellweger, A. Burg, N. Felber, and W. Fichtner, "K-Best MIMO detection VLSI architectures achieving up to $424 \mathrm{Mbps,"} \mathrm{in} \mathrm{Proc.}$ IEEE Int. Symp. Circuits and Systems, Kos, Greece, May21-24 2006, pp. 1151-1154.

[8] Z. Guo and P. Nilsson, "Algorithm and implementation of the K-best sphere decoding for MIMO detection," IEEE Journal on Select Areas in Communications, vol. 24, no. 3, pp. 491-503, March 2006.

[9] "M. G. Datasheet, "Catapult Synthesis," Mentor Graphics, Tech. Rep., 2008, http://www.mentor.com/products/esl/high_level_synthesis/ catapult_synthesis/index.cfm."

[10] J. Ketonen and M. Juntti, "SIC and K-best LSD receiver implementation for a MIMO-OFDM system," in Proc. European Sign. Proc. Conf., Lausanne, Switzerland, Aug. 25-29 2008.

[11] I. Collings, M. Butler, and M. McKay, "Low complexity receiver design for MIMO bit-interleaved coded modulation," in Proc. IEEE Int. Symp. Spread Spectrum Techniques and Applications, Sydney, Australia, Aug. 30 - Sep. 2 2004, pp. 1993-1997.

[12] M. Myllylä, J. Antikainen, M. Juntti, and J. Cavallaro, "The effect of LLR clipping to the complexity of list sphere detector algorithms," in Proc. Annual Asilomar Conf. Signals, Syst., Comp., Pacific Grove, USA, Nov. 4-7 2007.

[13] P. Bengough and S. Simmons, "Sorting-based VLSI architectures for the M-algorithm and T-algorithm trellis decoders," IEEE Transactions on Communications, vol. 43, no. 234, pp. 514 - 522, February 1995.

[14] Y. Sun, Y. Zhu, M. Goel, and J. Cavallaro, "Configurable and scalable high throughput turbo decoder architecture for multiple $4 \mathrm{G}$ wireless standards," in IEEE Int. Conf. on Application-specific Systems, Architectures and Processors (ASAP), Leuven, Belgium, Jul.2-4 2008, pp. 209-214. 